\title{
INTERNALIZACIÓN DE LA PAZ, MEDIANTE LAS COMPETENCIAS SOCIOAFECTIVAS EN NIÑOS Y NIÑAS DE PREESCOLAR EN NEIVA
}

\section{INTERNALIZATION OF PEACE THROUGH SOCIO-AFFECTIVE COMPETENCIES IN PRESCHOOL CHILDREN IN NEIVA}

\section{Emperatriz Perdomo Cruz ${ }^{1}$}

\section{Leidy Carolina Cuervo²}

\section{Jenifer Lorena Barrera Monje ${ }^{3}$}

\section{Eduardo Plazas Motta ${ }^{4}$}

$1 \quad$ Líder de la investigación. Licenciada en Pedagogía Infantil y Magíster en Educación, de la Universidad Surcolombiana en Neiva-Colombia, Doctoranda en Educación de la Universidad Americana de Europa en Cancún-México, Investigadora Grupo Paz desde la Paz de la USCO y Docente Universitaria y Docente de Preescolar. Orcid: https://orcid.org/0000-0002-3336472X $2 \quad$ Licenciada en Pedagogía Infantil, Especialista en Integración Educativa y Magister en Educación, de la Universidad Surcolombiana en Neiva-Colombia, Doctoranda en Educación de la Universidad Americana de Europa en Cancún-México, Coordinadora Grupo Paz desde la Paz de la USCO y Docente Universitaria. ORCID https://orcid.org/0000-0002-6927-0776.

$3 \quad$ Licenciada en Pedagogía Infantil, Especialista en Pedagogía, Especialista en Inclusión de la Universidad Surcolombiana en Neiva-Colombia, Magister y Doctoranda en Educación desde la Diversidad de la Universidad de Manizales en Manizales-Colombia, Investigadora Grupo Paz desde la Paz de la USCO, Docente Universitaria y Docente de Primaria. https:/l orcid.org/0000-0002-1179-2977

$4 \quad$ Licenciado en Administración Educativa y Especialista en Pedagogía, de la Universidad Surcolombiana en NeivaColombia, Especialista en Docencia Universitaria de la Universidad Cooperativa de Colombia en Neiva- Colombia, Magister en Educación de la Universidad del Valle en Cali-Colombia, Doctorando en Educación de la Universidad DEUSTO en Bilbao- España y Docente Universitario. ORCID iD https://orcid.org/ 0000-0002-4471-4481 


\section{RESUMEN}

Conseguir la paz ha sido el propósito esencial de todo ser humano a nivel mundial, con los años los conflictos han aumentado en todos los ámbitos de la sociedad y la solución mediática es la violencia. La investigación se realizó en la Institución Educativa Enrique Olaya Herrera de la ciudad de Neiva, en la que participó toda la comunidad educativa.

Como referente teórico, la Tesis de la Paz Interior, la Teoría de la Paz Imperfecta, (López, 2000, p. 46-49).Y la "Teoría de la trasmisión cultural" de (Bernstein, 1994). En coherencia con los objetivos propuestos el estudio fue de enfoque cualitativo, desde acciones como: elaboración teórica, revisión documental y caracterización de los contextos de interacción pedagógicos; fue de carácter: analítico, descriptivo, interpretativo y propositivo.

Los resultados abordaron importantes conclusiones y recomendaciones, se propuso empoderamiento conceptual, procedimental y actitudinal, se reconoció transformar las interacciones pedagógicas de los agentes. Se planteó construir relaciones menos violentas y solución pacífica de los conflictos. E implementar un currículo experiencial fundamentado en la pedagogía invisible.

PALABRAS CALVE: paz, internalización, competencias socioafectivas, conflicto y violencia.

\section{INICIANDO EL CAMINO HACIA LA INTERNALIZACIÓN DE LA PAZ}

La investigación se realizó en la Institución Educativa Enrique Olaya Herrera de la ciudad de Neiva, en la que participaron toda la comunidad educativa. Se trabajó con las siguientes variables cualitativas de análisis: paz, internalizacion, competencias socioafectivas, conflicto y violencia. El fenómeno de la violencia que ha venido aquejando al país durante los últimos años se ha acrecentado afectando a la población más vulnerable, por lo que la Escuela pública ha sido permeada por esta problemática, dado que allí se reciben estudiantes víctimas de la violencia.

Desde el nivel preescolar los niños y las niñas manifiestan las diversas formas de la violencia, entre las más reiterativas están la verbal y la física que vivencian en sus nichos familiares y del barrio. Al avanzar a los grados superiores esta problemática es de mayor potencia y frecuencia, incluso la utilización de armas convirtiéndose en una problemática social.

El propósito principal de este estudio fue que los niños y las niñas desde el nivel preescolar aminoraran los comportamientos y actitudes de violencia, e internalizaran las virtudes de la paz mediada por las competencias socioafectivas. Donde la escuela suscite en sus interacciones pedagógicas, las vivencias de estas virtudes: el dominio propio, la bondad, la justicia, la esperanza, el afecto y la alegría. En Colombia la educación preescolar es la responsable de la formación de los niños y las niñas con bases sólidas, creadora de valores y aptitudes para ser competentes y aprender a convivir con otros.

En el Capítulo 1. Inicialmente, se hizo necesario realizar un análisis del problema de violencia a nivel mundial, nacional, local e institucional. Además, se hizo un recorrido recopilando todas las acciones hacia la paz, a nivel macro, mezo y micro, de orden gubernamental y privado partiendo de los objetivos.

En el Capítulo 2. Se abordó el referente teórico, la "Tesis de la Paz Interior", los fundamentos epistemológicos de la "Teoría de la Paz Imperfecta" (López, 2000, p. 46-49).Y la "Teoría de la Transmisión Cultural” (Bernstein, 1994), fueron básicas para la comprensión de los estudios sobre la internalización de las virtudes de la de la paz. 
En el Capítulo 3. Se trabajó el diseño de la investigación, se fundamentó desde la elaboración teórica, la revisión documental y caracterización de los contextos pedagógicos, fue de carácter analítico, descriptivo, interpretativo y propositivo; con un enfoque cualitativo. (Briones, 1998).

Y en el Capítulo 4. Se abordaron importantes conclusiones y recomendaciones, se recomendó empoderar conceptual, procedimental y actitudinal a todos los. Se reconoció la importancia de transformar las interacciones de los actores. Se propuso rescatar espacios para la paz, donde se faciliten escenarios de diálogo y resolución pacífica de conflicto. E implementar un currículo experiencial desde la pedagogía invisible.

\section{PLANTEAMIENTO DEL PROBLEMA.}

La violencia verbal, física, psicológica y emocional son manifestaciones de la violencia escolar, se presentan constantemente en las Instituciones Educativas, debido a diversas situaciones como: discriminación cultural, racial, social, religiosa, de clase, entre otros. Es necesario que los niños y las niñas internalicen los comportamientos y actitudes que construyen la paz, en este proceso diversos sectores piensan que la violencia es un ambiente común y natural en la etapa evolutiva en la Escuela, por tanto pasan por alto la conducta del violento, el violentado y el espectador.

En este punto, es preciso entender que la violencia escolar es un problema mundial que puede ocurrir en cualquier tipo de Escuela, sea pública o privada, en seres humanos en diferentes espacios donde existe un débil y un atacante. No obstante, hacer referencia de una persona débil no alude exclusivamente a la dimensión física, además, de esa connotación, también, la dimensión emocional. Una persona que domina sus sentimientos y emociones, es una persona capaz de manejar con cierto grado de confianza, la mayoría de las situaciones adversas en las que se pueda ver involucrado. Esa persona no flota en un mar de emociones, sino que es consciente de los sentimientos que experimenta y sabe cómo afrontarlos. KlerkWyer \& Le Roux (2012).

Una de principales causas de la violencia escolar podrían ser: por la falta de conocimientos y la falta de formación en las competencias socioafectivas para la paz. La primera, tiene que ver con el papel que debe lograr la educación como actor en la adquisición del conocimiento pertinente para la formación de competencias socioafectivas. De la misma manera, la paz no se agota en lo escolar, quiere esto decir, que la escuela no es el único escenario de formación, pues recae en la familia y sociedad contribuir a la internalización de la paz.

\section{PREGUNTA PROBLEMATIZADORA.}

¿En qué sentido son coherentes y pertinentes las competencias socioafectivas como mediadoras para la internalización de la paz, en los niños y las niñas de preescolar en la institución educativa Enrique Olaya Herrera sede Central y Camelias, en la Comuna Diez de la Ciudad de Neiva?

\section{PROPÓSITOS PARA INTERNALIZAR LAS VIRTUDES DE LA PAZ EN LA COMUNIDAD EDUCATIVA}

Objetivo General. Analizar, identificar y describir críticamente, la pertinencia de las competencias socioafectivas como mediadoras para la internalización de la paz, en los niños y las niñas del nivel preescolar de la Institución Educativa Enrique Olaya Herrera Sede Central y Camelias, en la Comuna Diez de la ciudad de Neiva. 


\section{OBJETIVOS ESPECÍFICOS}

- Identificar las situaciones de conflicto que generan violencia que se presentan en los contextos de interaccion pedagógicos de la Escuela, entre los niños y las niñas del nivel preescolar, de la Institución Educativa Enrique Olaya Herrera Sede Central y Camelias, en la Comuna Diez de la ciudad de Neiva.

- Determinar la pertinencia de las competencias socioafectivas como mediadoras de la internalización de la paz desde las interacciones pedagógicas de la Escuela en coherencia con las etapas y características de desarrollo y aprendizaje de los niños y las niñas del nivel preescolar, de la Institución Educativa Enrique Olaya Herrera Sede Central y Camelias, en la Comuna Diez de la ciudad de Neiva.

- Caracterizar la pertinencia de las competencias socioafectivas como mediadoras de la internalización de la paz desde las interacciones pedagógicas en la familia y el barrio, de acuerdo con las etapas y particularidades del desarrollo de los niños y las niñas del nivel preescolar, de la Institución Educativa Enrique Olaya Herrera Sede Central y Camelias, en la Comuna Diez de la ciudad de Neiva.

- Proponer algunas recomendaciones, que garanticen la pertinencia de las competencias socioafectivas como mediadoras de la internalización de las virtudes de la paz tanto en los contextos de interaccion pedagógicos de la Escuela, la familia y el barrio, de los niños y las niñas del nivel preescolar, de la Institución Educativa Enrique Olaya Herrera Sede Central y Camelias, en la Comuna Diez de la ciudad de Neiva.

\section{IMPORTANCIA DE LA INTERNALIZACIÓN DE LAS VIRTUDES DE LA PAZ}

Con el interés de tomar una postura clara, frente a comportamientos y actitudes, de cualquier forma de violencia, que obstaculiza el desarrollo socioafectivo. El estudio busco sensibilizar sobre el conocimiento de esta problemática, para prevenir estas prácticas de violencia y promover la internalización de las virtudes de la paz.

A nivel internacional, los eventos en cuanto a la problemática de la violencia escolar, han permitido investigaciones, se tienen cifras que se plantean normas y programas de prevención. Para ilustrar una investigación en España de la pedagoga Rodríguez (2004), arrojó resultados alarmantes $48 \%$ de los estudiantes españoles entre 9 y 14 años ha sufrido o sufre algún tipo de agresión por parte de algún compañero, más del $50 \%$ agresión psicológica y el $20 \%$ agresión física.

En Colombia la situación comenzó a tener auge luego de sucesos surgidos en instituciones educativas del país por lo que la Corte ordenó al MEN (Ministerios de Educación Nacional) e ICBF( Instituto Colombiano de Bienestar Familiar) implementar políticas en los manuales de convivencia de todas las instituciones del país. Se sancionó la (Ley 1620 de 2013).

En Neiva en el año 2017, con el propósito de conocer la real situación problemática que aqueja a la comunidad educativa que directamente afecta la convivencia dentro de las instituciones educativas, se consideró pertinente tipificar estos comportamientos objeto de análisis de este estudio, se pudo concluir que los comportamientos que afectan la convivencia en las instituciones educativas en orden de mayor frecuencia fueron: las agresiones con 18.015 casos, le siguen las comportamentales con 9.049 casos.

Hay que concebir que la violencia escolar, es una de principales problemáticas de la educación infantil, por lo tanto, es necesario promover la paz tanto en el contexto de la Escuela como en la familia y la comunidad. 


\section{DESDE LA LUZ QUE GUÍA EL CAMINO HACIA LA INTERNALIZACIÓN DE LAS VIRTUDES PARA LA PAZ}

\section{MARCO TEÓRICO.}

La Tesis de la Paz Interior, se considera como un estado interior que trasforma a cada persona, que se exterioriza hacia sus semejantes por medio de comportamientos y actitudes. Que construyen la paz día a día desde sus interacciones, se sustenta en las virtudes de: el dominio propio, la bondad, la justicia, la esperanza, el afecto y la alegría.

La Teoría de la Paz Imperfecta, por lo tanto comprender cómo una cualidad esencial de la Paz es promover que los conflictos sean gestionados, transformados o regulados de manera pacífica los conflictos atravesando actores, espacios y tiempos, es vital. (Muñoz Muñoz, 2004, p.28).

Y la "Teoría de la trasmisión cultural" de (Bernstein, 1994), se señala el estudio sobre las relaciones de poder y los principios de control que generan, distribuyen, reproducen y legitiman las formas específicas de dominación, en los diversos contextos de interacción pedagógica de la Escuela.

\section{MARCO CONCEPTUAL.}

Estos conceptos permitieron abordar el estudio de la internalización de la Paz, en los contextos pedagógicos de interacción.

\section{CONCEPTO DE PAZ.}

Paz Interior, se considera como un estado interior que trasforma a cada persona, que se exterioriza hacia sus semejantes por medio de comportamientos y actitudes. Que construyen la paz día a día desde sus interacciones, se sustenta en las virtudes de: el dominio propio, la bondad, la justicia, la esperanza, el afecto y la alegría.
Por lo tanto comprender cómo una cualidad esencial de la Paz es promover que los conflictos sean gestionados, transformados o regulados de manera pacífica los conflictos atravesando actores, espacios y tiempos, es vital. Es más creo que estas interacciones de los espacios donde se potencia la regulación pacífica de los conflictos es una cualidad de esta Paz imperfecta. (Muñoz Muñoz, 2004, p. 28).

\section{CONCEPTO DE INTERNALIZACIÓN.}

La transformación de un proceso interpersonal en un proceso intrapersonal, es el resultado de una prolongada serie de sucesos evolutivos. La transformación de un proceso interpersonal en un proceso intrapersonal. (Vigotsky, 1987, p. 5).

\section{CONCEPTO DE COMPETENCIAS SOCIOAFECTIVAS.}

Se relaciona con el comportamiento, actitud y aptitud de los seres humanos en el contexto social donde conviven, intervienen en el desarrollo de sus valores y virtudes, es decir, lo axiológico, a partir del saber conocer, saber-hacer y saberser, desde las habilidades interpersonales e intrapersonales. (Perdomo Cruz, 2018).

\section{CONCEPTO DE CONFLICTO.}

Desde la perspectiva de los pacifistas los conflictos son una de las bases teóricas y prácticas de toda la actividad humana, por ello intentamos comprenderlos lo mejor posible y, a partir de ahí, proponer técnicas y metodologías de regulación y prevención de los mismos. (Muñoz Muñoz, 2004, p.143).

\section{CONCEPTO DE VIOLENCIA.}

La violencia es una acción injusta con que se ofende o perjudica a alguien, está presente cuando los seres humanos se ven influidos de tal manera que sus realizaciones efectivas, somáticas y mentales están por debajo de sus 
realizaciones potenciales. (Martin, 2004 p.224229).

\section{CONCEPTO PEDAGOGÍA INVISIBLE}

La práctica pedagógica en principio es invisible para el adquiriente, fundamentalmente porque este llena el espacio pedagógico. Su centro de interés está constituido por los procedimientos internos del adquiriente (Cognitivos, lingüísticos, afectivos, motivacionales) a consecuencia de los cuales se crea y experimenta un texto. (Bernstein, 1998, p.80).

\section{MARCO LEGAL}

\section{MARCO LEGAL INTERNACIONAL.}

Resolución 53/243 de (1999). Declaración y Programa de Acción para una Cultura de Paz. Asamblea General de las Naciones Unidas.

Proclama solemnemente que con el fin de que los Gobiernos, las organizaciones internacionales y la sociedad civil puedan orientar sus actividades por sus disposiciones a fin de promover $y$ fortalecer una cultura de paz en el nuevo milenio: Declara en su Artículo 1. Una cultura de paz es un conjunto de valores, actitudes, tradiciones, comportamientos y estilos de vida basados en:

Resolución 71/189 de (2016).Declaración sobre el Derecho a la Paz. Asamblea General de las Naciones Unidas.

Invita solemnemente a todos los interesados a que en sus actividades, se guíen por el reconocimiento de lo extremadamente importante que es practicar la tolerancia, el diálogo, la cooperación y la solidaridad entre todos los seres humanos, los pueblos y las naciones del mundo como medio para promover la paz. Para lo cual las generaciones actuales deben velar por que tanto ellas mismas como las generaciones futuras aprendan a convivir en paz, con la aspiración máxima de salvar a las generaciones futuras del flagelo de la guerra. Declara lo siguiente en su Artículo 1. Toda persona tiene derecho a disfrutar de la paz de tal manera que se promuevan y protejan todos los derechos humanos y se alcance plenamente el desarrollo. Objetivo $16 \mathrm{Paz}$, justicia e instituciones sólidas. Promover sociedades pacíficas e inclusivas para el desarrollo sostenible, facilitar el acceso a la justicia para todos y crear instituciones eficaces, responsables e inclusivas a todos los niveles.

\section{OBJETIVOS DE DESARROLLO SOSTENIBLE (2016).}

Por la Asamblea de las Naciones Unidas, los objetivos de desarrollo sostenible son 17 asociados a la Agenda 2030 para el Desarrollo Sostenible, aprobada en septiembre de 2015 por la Asamblea General de las Naciones Unidas, establece una visión transformadora hacia la sostenibilidad económica, social y ambiental de los 193 Estados Miembros que la suscribieron y será la guía de referencia para el trabajo de la institución en pos de esta visión durante los próximos 1 Constitución Política de Colombia de (1991). Dado que la Constitución Política de Colombia se constituye en la ley Magna, que protege y promueve los derechos fundamentales de los colombianos, especialmente las de los niños y las niñas en la primera infancia, por lo tanto en los artículos que a continuación se mencionan, se habla de específicamente que se promueve la protección contra toda forma de violencia. Artículo 67. La educación formará al colombiano en el respeto a los derechos humanos, a la paz y a la democracia; y en la práctica del trabajo y la recreación, para el mejoramiento cultural, científico, tecnológico y para la protección del ambiente.

\section{MARCO LEGAL NACIONAL}

Ley 115 de (1994). Ley General de Educación, por la cual se expide la Ley General de Educación. El Congreso de la República de Colombia. Bogotá-Colombia. Artículo $5^{\circ}$. Fines 
de la educación. De conformidad con el artículo 67 de la Constitución Política, la educación se desarrollará atendiendo a los siguientes fines: 2 . La formación en el respeto a la vida y a los demás derechos humanos, a la paz, a los principios democráticos, de convivencia, pluralismo, justicia, solidaridad y equidad, así como en el ejercicio de la tolerancia y de la libertad.

Ley 1620 de (2013). Ley de Convivencia Escolar, Sistema Nacional de Convivencia Escolar y Formación para el Ejercicio de los Derechos Humanos, Sexuales y Reproductivos y la Prevención y Mitigación de la Violencia Escolar. El objetivo principal es contribuir a la formación de ciudadanos activos que aporten a la construcción de una sociedad democrática, participativa, pluralista e intercultural, en concordancia con el mandato constitucional y la Ley General de Educación -Ley 115 de 1994. Mediante la creación del sistema nacional de convivencia escolar y formación para los derechos humanos, la educación para la sexualidad y la prevención y mitigación de la violencia escolar, que promueva y fortalezca la formación ciudadana y el ejercicio de los derechos humanos, sexuales y reproductivos de los estudiantes; de los niveles educativos de preescolar, básica y media y prevenga y mitigue la violencia escolar y el embarazo en la adolescencia.

Ley 1732 de (2014) Por la cual se establece la cátedra de la paz en todas las instituciones educativas del país.

Artículo $1^{\circ}$. Con el fin de garantizar la creación y el fortalecimiento de una cultura de paz en Colombia, establézcase la Cátedra de la $\mathrm{Paz}$ en todas las instituciones educativas de preescolar, básica y media como una asignatura independiente.

Ley 1804 de (2016)."Por La Cual se Establece la Política de Estado para el Desarrollo Integral de la Primera Infancia de Cero A Siempre y se Dictan otras Disposiciones". La presente iniciativa legislativa tiene el propósito de establecer la Política de Estado para el Desarrollo Integral de la Primera Infancia de Cero a Siempre, la cual sienta las bases conceptuales, técnicas y de gestión para garantizar el desarrollo integral, en el marco de la Doctrina de la Protección Integral. Con ello busca fortalecer el marco institucional para el reconocimiento, la protección y la garantía de los derechos de las mujeres gestantes y de los niños y las niñas de cero a seis años de edad, así como la materialización del Estado Social de Derecho.

\section{EN LA RUTA QUE LLEVA A LA INTERNALIZACIÓN DE LAS VIRTUDES DE LA PAZ}

Naturaleza de la Investigación. Esta investigación, se fundamentó a partir de la integración de acciones relacionadas con la elaboración teórica, desde la revisión documental y la caracterización de los diversos contextos pedagógicos. Fue de carácter: analítico, descriptivo, interpretativo y propositivo, tiene un enfoque cualitativo (Briones, 1998).

\section{VARIABLES.}

Fueron de carácter cualitativo, dado que la particularidad que midieron de la unidad de análisis o una cualidad, cada una de ellas están relacionadas directamente con el referente empírico y conceptual de la investigación y permitió leer la realidad estudiada acerca de la internalización de las virtudes de la paz mediada por la competencias socioafectivas.

Las variables que se tuvieron en cuenta para el desarrollo de esta investigación, fueron definidas conceptualmente y están directamente coherentes con las que sustentan el referente teórico, conceptual y empírico de la misma. Variables: paz, internalizacion, competencias socioafectivas, conflicto y violencia. (Hernández 
Sampieri, Fernández Collado \& Baptista Lucio, 2010, p. 93).

\section{POBLACIÓN Y MUESTRA}

Dentro de la población de agentes con los que se ejecutó la investigación de la Institución Educativa Enrique Olaya Herrera se considera. Ludewig (1998).

\section{LA POBLACIÓN}

Estuvo constituida por 4.555 agentes, que pertenecen a la institución educativa Enrique Olaya Herrera del sector público de la zona urbana de la ciudad de Neiva, segregados en directivos docentes, docentes, estudiantes y los padres de familia de los estudiantes. García Muñoz (2005).

\section{LA MUESTRA}

Fue intencional, el $100 \%$ de los directivos docentes, el $100 \%$ de los docentes del nivel preescolar, el $100 \%$ de los estudiantes del nivel preescolar, y aproximadamente el $45 \%$ de los padres de familia de los estudiantes del nivel preescolar y estuvo constituida por 346 sujetos.

\section{INSTRUMENTOS DE RECOLECCIÓN DE LA INFORMACIÓN.}

Para efecto de la recolección de los datos, se elaboraron cuestionarios, entrevistas a profundidad y registros de observación, para dar cumplimiento a los objetivos propuestos, sobre el estudio de la internalización de las virtudes de la paz mediadas por las competencias socioafectivas, para la prevención de la violencia. Fàbregues, Meneses, Rodríguez, Gómez \& Paré. (2016).

EI cuestionario Como elemento esencial, se consideró pertinente conocer la opinión de los docentes y los padres de familia, frente al desarrollo, estructuración e internalizacion la paz mediada por las competencias socioafectivas en los niños y niñas en el nivel de preescolar. Se realizó el diligenciamiento de los cuestionarios por parte de los siete (7) docentes de preescolar. El otro cuestionario se aplicó a setenta y siete (77) padres de familia. Hernández, Fernández \& Baptista (2010) (citado por Brace, 2008).

La entrevista. Se realizaron a cinco (5) directivos docentes, (1) rectora, (3) coordinadores y (1) orientadora escolar. Se consideró como un recurso importante dado que se pretendió conocer las opiniones y concepciones acerca de la importancia de las competencias socioafectivas como mediadoras para la internalización de la paz, y opiniones acerca de la normatividad del (MEN). Hernández et al (2010).

El registro de observación. Se aplicó veinte y cuatro (24) veces en total de las veinte y ocho (28) veces previstas, cuatro (4) por aula, a 143 niños y niñas de los 163 previstos en total, ubicados en seis (6) aulas. Solo una docente no permitió que se aplicara registro de observación en su aula. Hernández et al (2010).

\section{PROCESAMIENTO DE LA INFORMACIÓN DESDE LOS ARGUMENTOS DE LOS AGENTES DE LA COMUNIDAD EDUCATIVA EN CONCERNIENTES A LA INTERNALIZACIÓN DE LA PAZ}

El procesamiento de la información de las preguntas objeto de análisis, que correspondían a cada una de las variables cualitativas mediante el programa NVIVO V. 12.0. Se crearon unos Nodos o variables de análisis de los conceptos que los docentes, padres de familia y directivos docentes respondieron en cada pregunta, los cuales fueron organizados a través de la técnica de Codificación Abierta; que es un proceso analítico por medio del cual se identifican los conceptos y se descubren en los datos sus propiedades y dimensiones. Codificación abierta: el proceso analítico por medio del cual se identifican los conceptos y se descubren en los datos sus propiedades y dimensiones. 
Fenómenos: ideas centrales en los datos, representadas como conceptos.

Conceptos: basamentos fundamentales de la teoría.

Categorías: conceptos que representan fenómenos.

Propiedades: características de una categoría, cuya delineación la define y le da significado.

Dimensiones: escala en la cual varían las propiedades generales de una categoría, y que le da especificaciones a la categoría y variaciones a la teoría.

Subcategorías: conceptos que pertenecen a una categoría, que le dan claridad adicional y especificidad. (Strauss \& Corbin, 2002, p. 110).

Este procedimiento se estructuro tanto en las respuestas del cuestionario de docentes y padres de familia y a la entrevista de directivos docentes. A continuación, se detalla la organización que se estructuro en el programa NVIVO. Por cada Nodo se desarrollaron unos SubNodos, para organizar el análisis del contenido de los conceptos. "Nodos o categorías de análisis, y codificación de los documentos. Como dijimos, los nodos son nuestras categorías de análisis. Podemos crearlos mediante dos maneras: a) los hemos acordado previamente, ya sea desde el marco teórico, equipo de investigación, etc., b) los podemos ir creando conforme vamos leyendo los relatos, entrevistas, grupos, o textos que deseemos analizar. Ambos procedimientos también se pueden realizar en el programa." (Meneses Falcón, C 2019, p. 16).

Mediante análisis estadístico, los datos cuantitativos se consolidaron a través de una matriz organizada en Microsoft office Excel, paquete de versión 2010. Los datos de las respuestas de las preguntas sociodemográficas y de los registros de observación, fueron analizaos estadísticamente, utilizando el software SPSS V 12.0.

Se exploraron y depuraron para contrastar la normalidad de los datos mediante las pruebas de bondad Kolomogorv-Smirnov, con factor de corrección de significación Lilliefors para ver si cumplían las distribuciones normales. (RomeroSaldaña, M 2016, p. 36 Y 38)

Se aplicó la estadística descriptiva el análisis con frecuencias relativas y absolutas, se crearon variables dicotómicas que representaron los valores límites de cada variable, previamente determinados de acuerdo con los valores normales de la población, en las respuestas delas preguntas de las variables sociodemográficas, de igual forma se hizo el procesamientos de la información con las respuestas de los ítems de los registros de observación. (Castañeda, Cabrera, \& Navarro. 2010, p. 26).Y (Álvarez González, p. 3).

\section{HALLAZGOS ACERCA DE LAS COMPETENCIAS SOCIOAFECTIVAS PARA INTERNALIZAR LA PAZ EN LOS DIVERSOS CONTEXTOS DE INTERACCIÓN PEDAGÓGICA DEL PREESCOLAR}

El presente estudio realizado tiene las siguientes conclusiones:

-Se pudo demostrar que la gran mayoría de agentes manejan un código restringido frente al concepto de paz, donde la asumen como negativa o ausencia de violencia y como positiva en armonía total, solo muy pocos agentes reconocen la paz como un estado interior. La Tesis de la Paz Interior y la Teoría de la Paz Imperfecta. (López Muños, 2001, p. 38).

-Se demostró que los agentes manejan un código restringido en cuanto al concepto de las normas del Ministerio de Educación Nacional. Ley 1620 de (2013). 
-Respecto a la Cátedra de la Paz, no es coherente la respuesta de los agentes educativos con los referentes estipulados en el P.E.I y practica pedagógica, no se ha implementado. Ley 1732 de (2014).

- Se concluyó que todos los agentes del estudio manejan un código elaborado frente al concepto de internalizacion. (Vigotsky, 1978, p. 94).

-Se evidencio que los espacios físicos legítimos que promueven las competencias socioafectivas para internalizar la paz, son solo los de la institución. (Zurbano Días de Cerio, 2001, p. 17 y 20).

-Se pudo concluir que la institución educativa carece de recursos didácticos adecuados a la étapa del desarrollo de los niños y las niñas de este nivel, para internalizar las virtudes de la paz. (Zurbano Días de Cerio, 2001, p. 47).

-Se concluye que el ambiente físico y afectivo del aula no es muy motivador para internalizar la paz desde las competencias socioafectivas en los niños y las niñas. (Bernstein, 1998, p. 76).

-Se evidencio que los agentes participantes del estudio manejan un código elaborado frente al concepto de competencias socioafectivas.(Transversales. Educación para la paz, M.E.C., 1992, et al Zurbano Días de Cerio, p. 21, 1998).

-Se concluyó que la forma de organización de los niños, las niñas y la docente en el aula al momento de trabajar las competencias socioafectivas para la internalización de la paz, expresa una concepción tradicional y jerárquica del proceso de formación. (Bernstein, p. 37, 1994).
-Se demostró que desde el fomento de las competencias socioafectivas para la internalización de la paz,se abordan desde una pedagogía visible, donde los conocimientos priman sobre los procedimientos y actitudes. Chaux (2012, p.78).).

-Se pudo concluir que la naturaleza de la práctica pedagógica entre las docentes y los estudiantes, se caracteriza por ser vertical se diferencia rol de cada uno de ellos, y manejan códigos restringidos. (Bernstein, 1998, p. 101-116).

-Se demostró que es imperceptible el trabajo de las virtudes para la internalización de la paz, desde las competencias socioafectivas en el aula, generando códigos restringidos de su contesto local, que predominan sobre los códigos elaborados del contexto universal. (Bernstein, 1998, p.7).

-Se ha demostrado que los padres de familia manejan un código restringido, en cuanto a la promoción de las competencias socioafectivas para la internalización de la paz, carecen de coherencia en la acción. (De Cerio, 1998, p. 13).

-Se evidencio que todos los agentes asumen que el conflicto son situaciones inherentes a la vida diaria, coherente con la cosmovisión que se tiene en el país acerca de este "conflicto armado". Generando una concepción tradicional negativa, derivado de un código restringido. (Muñoz, 2004, p. 143).

-Se demostró que no existe coherencia entre lo que los padres de familia argumentan frente a la solución de conflictos en la familia; con lo que demuestran los niños y las niñas en la escuela. (Muñoz, p. 163, 2004). 
-Se concluyó que todos los niños y las niñas asumen los conflictos como negativos, es decir, cuando estos se presentan en la escuela los solucionan mediante la violencia. (Muñoz, p. 163, 2004).

-Se ha concluido que todos los agentes manejan un código elaborado frente al concepto de violencia, lo relacionan el contexto universal y el ambiente local. (Martín Morillas, 2004, p. 224).

-Se demostró que todos los padres de familia manejan un código elaborado acerca de las formas de violencia, lo relacionan con el contexto universal y su ambiente local. (Muñoz \& Molina Rueda, 2004, p. 253, 256, $257,262,269)$.

-Se demostró que las familias viven en condiciones de vulnerabilidad, que generan otras formas de violencia hacia ellos, como el desplazamiento, la pobreza, el hambre, el hacinamiento etc. (Muñoz \& Molina Rueda, 2004, p.257).

\section{RECOMENDACIONES PARA INTERNALIZAR LAS VIRTUDES DE LA PAZ EN LOS NICHOS DE INTERACCIÓN PEDAGÓGICA DEL PREESCOLAR}

El presente estudio plantea las siguientes recomendaciones:

-Empoderar teórica y conceptualmente a los agentes de la comunidad educativa en los postulados de este estudio referente al concepto de paz. Tesis de la Paz Interior y la Teoría de la Paz Imperfecta. (López Muños, 2001, p. 38).

-Implementar de forma trasversal en el currículo la Cátedra de la Paz, conocimientos, procedimientos y actitudes. Ley 1732 de (2014).
-Que las docentes promuevan formas de comunicación más invisibles-horizontales con una enmarcación débil con los niños y las niñas, donde el acto pedagógico referente la paz este centrado en el estudiante. (Bernstein, 1998, p. 72 y 73).

-Que la Institución educativa promueva otros espacios físicos fuera de la Escuela, donde los niños y las niñas lleven a la práctica las virtudes para internalizar la paz, desde las competencias socioafectivas. (Zurbano Días de Cerio, 2001, p. 17 y 20).

-Se recomienda que la institución educativa implemente recursos didácticos variados y pertinentes, además, que las docentes los manejen de forma significativa y motivadora. (Zurbano Díaz de Cerio, 2001. P. 147).

-Las docentes deben promover una formación menos jerarquizada con identidades menos diferenciadas entre ellos, donde utilicen más estrategias enmarcadas en todas las actividades rectoras, que motiven a los niños y a las niñas a participar, disfrutar y a aprender. (Bernstein, p. 37, 1994).

-Los docentes deben promover ambientes físicos y afectivos adecuados, tener en cuenta las reglas de secuencia, es decir; las etapas de evolutivas desarrollo y las características que se presentan los niños y las niñas en cada una de ellas. (Bernstein, 1994, p. 76).

-Fortalecer las prácticas pedagógicas universales sobre las virtudes para internalizar la paz en la Escuela; para que predominen sobre las prácticas locales de violencia. (Bernstein, 1998, p. 72 y 73).

-Desde la Teoría de la Transmisión Cultural, implementar la pedagogía invisible donde solo el transmisor-docente conoce las reglas discursivas, el adquiriente-estudiante llena 
el espacio pedagógico, haciendo hincapié en la adquisición de la competencia. (Bernstein, 1998, p. 80).

-Trabajar desde un currículo experiencial los contenidos conceptuales, procedimentales y actitudinales, para internalizar las virtudes de la paz desde las competencias socioafectivas. (Chaux ,2012 p. 78) y (Zurbano Días de Cerio, p. 13, 2001).

-Las docentes deben fomentar contextos más universales, que promuevan la internalización de las virtudes de la paz, que dominen sobre el contexto local de violencia de los niños y las niñas. (Bernstein, p. 102, 1994).

-Se ha de promover una práctica pedagógica en la forma de comunicación entre las docentes, los niños y las niñas, que se caracterice por ser horizontal y se manejen unos códigos elaborados. (Bernstein, 1994. p. 47,101 y 116).

-Empoderar teórica y conceptualmente a los agentes de la comunidad educativa en los postulados de este estudio referente al concepto de conflicto. Teoría de la Paz Imperfecta. (López Muños, 2001, p. 38).

-Es necesario que los agentes educativos manejen más estrategias de resolución a los conflictos en la escuela. Paz Imperfecta. (Muñoz, p. 163, 2004).

-Que la institución educativa empodere a los padres de padres de familia frente a la solución de conflictos dentro de la familia y sus vecinos que manejen la coherencia en la acción. (Muñoz, p. 163, 2004).

-Empoderar teórica y conceptualmente a los agentes de la comunidad educativa en los postulados de este estudio referente al concepto de violencia, dado que a menudo lo confunden con el de conflicto. Teoría de la Paz Imperfecta. (Martín Morillas, 2004, p. 224).

-Propender porque las prácticas pedagógicas universales, sobre las virtudes para internalizar la paz, ejercen dominancia sobre las prácticas pedagógicas violentas de la familia y el barrio. (Bernstein, 1998, p. 101-116).

-Motivar a las familias para que se beneficien de los programas que ofrece el Estado en la institución educativa, para mitigar las manifestaciones de violencia que las afecta (vulnerabilidad, simbólica, desplazamiento, pobreza, hambre, hacinamiento etc). Tesis de la Paz Interior y la Paz imperfecta. (Muñoz \& Molina Rueda, 2004, p.257).

\section{BIBLIOGRAFÍA}

$\begin{array}{ccc}\text { Álvarez González Francisco ( } & \text { ).Estadística } \\ \text { descriptiva. } & \text { Métodos } & \text { Estadísticos }\end{array}$ Aplicados a las Auditorías Socio laborales. Recuperado de https://www.academia. edu/29050852/Estad\%C3\%ADstica_ descriptiva_F._\%C3\%81lvarez_-1_ E S T A D \% C $3 \% 8$ D S I C A DESCRIPTIVA_M\%C $3 \%$ A 9 todos_ Estad\%C3\%ADsticos_Aplicados_a_las_ Auditor\%C3\%ADas_Sociolaborales.

Bernstein, B. (1994). La Estructura del Discurso Pedagógico. Clases, Códigos y Control. Vol. 4. Códigos, modalidades y proceso de reproducción cultural: un modelo. Pablo Manzano. Madrid: Morata $2^{\mathrm{a}}$ edición.

Bolaños Carmona, J (2005). Estadística descriptiva de una variable.Departamento de Estadística e I.O. Facultad de Biblioteconomía y Documentación. Universidad de Granada. Recuperado de https://www.ugr.es > rruizb > cognosfera > sala_de_estudio > Estadistica CognoSfera - www.ugr.es/local/rruizb/ cognosfera. 
Briones, G. (1998). La Investigación Social y Educativa. La Investigación en el aula y en la escuela. Edición del convenio Andrés Bello. Unidad Editorial. Editorial Gente Nueva. . Bogotá, Colombia.

Castañeda, M, Cabrera, A \& Navarro, Y. (2010). Procesamiento de datos y análisis estadísticos utilizando SPSS. Un libro práctico para investigadores y administradores educativos. Pontificia Universidad Católica de Rio Grande do Sul. EDIPUCRS. Porto Alegre- Brasil. Recuperado de http://www. pucrs.br/edipucrs/spss.pdf

Constitución Política de Colombia. (1994). Normatividad. Editado por: Corte Constitucional, Consejo Superior de la Judicatura, Centro de Documentación Judicial -CENDOJ y Biblioteca Enrique Low Murtra- BELM. Recuperado de http:// www.corteconstitucional.gov.co/inicio/ Constitucion $\% 20$ politica $\% 20$ de $\% 20$ Colombia.pdf.

Hernández Sampieri R, Fernández Collado C \& Baptista Lucio M P (2010). Metodología de la Investigación. McGraw-Hill / Interamericana Editores, S.A. DE C.V. México D.F. México. Recuperado de: www.FreeLibros.com.

Ley 115 de (1994). "Por la cual se expide la ley general de educación". El Congreso de la República de Colombia. Bogotá. Colombia.

Ley 1620 de (2013) "Por La Cual se Crea el Sistema Nacional de Convivencia Escolar y Formación para El Ejercicio de los Derechos Humanos, la Educación para la Sexualidad y la Prevención y Mitigación de la Violencia Escolar". Ministerio de Educación Nacional Colombia. Bogotá D.C. Colombia.

Ley 1732 de (2014) "Por la cual se establece la cátedra de la paz en todas las instituciones educativas del país" El Congreso de Colombia. Bogotá. Colombia.
Ley 1804 de. (2016). "Por la Cual se Establece la Política de Estado para el Desarrollo Integral de la Primera Infancia de Cero a Siempre y se Dictan otras Disposiciones". Ministerio de Educación Nacional Colombia. Bogotá D.C. Colombia.

Meneses Falcón, Carmen. (2019). Apuntes para trabajar con NVIVO 12 PLUS. Programa de Doctorado en Competitividad Empresarial y Territorial, Innovación y Sostenibilidad. Asignaturas: Métodos de Investigación Cualitativa. Universidad Pontificia Comillas Madrid, España. Recuperado de https:// riuma.uma.es/xmlui/handle/10630/17598.

Muñoz Muñoz, F.A \& Molina Rueda, B (2004). Manual de paz y conflictos. Editores Universidad de Granada. España. Recuperado de https://dialnet.unirioja.es/ servlet/libro?codigo=6645. Consulta: $(12 \mathrm{de}$ enero de 2016).

Muñoz, F. A \& Molina Rueda, B (2009). Una Cultura de Paz compleja y conflictiva. La búsqueda de equilibrios dinámicos. Revista paz y conflictos. N.3. Instituto de Paz y Conflictos de la Universidad de Granada. Granada. España. Recuperado de http:// www.ugr.es/ revpaz/articulos/rpc_n3_2010_ art3.pdf. Consulta: (20 de marazo de 2018).

Naciones Unidas (2016). Agenda 2030 y los objetivos de desarrollo sostenible una oportunidad para América Latina y el Caribe. Comisión Económica para América Latina y el Caribe (CEPAL). Santiago de Chile. Recuperado de http://www.sela. org/media/2262361/agenda-2030-y-losobjetivos-de-desarrollo-sostenible.pdf.

Resolución 53/243 (1999). Resoluciones Aprobadas por la Asamblea General de las Naciones Unidas. Declaración y Programa de Acción para una Cultura de Paz. Asamblea General de las Naciones Unidas. Aprobada 
el 6 de octubre de 1999. www.un.org/es/ documents/ag/res/33/ares33.htm. Consulta: (15 de noviembre de 2017).

Resolución 71/189. (2016). Resoluciones Asamblea General de las Naciones Unidas Declaración sobre el Derecho a la Paz. Aprobada por la Asamblea General el 19 de diciembre de 2016. Recuperado de: http://www.un.org/es/documents/index.html. Consulta: (15 de noviembre de 2017).

Romero-Saldaña, Manuel (2016), Metodología de la investigación. Pruebas de bondad de ajuste a una distribución normal. Revista Enfermería del Trabajo 2016; 6:3 (105114). Revista Julio 2016: Maquetación 1 03/09/2016 20:47 Página 39.

Trigueros Cervantes, C. Rivera García, E \& Rivera Trigueros I. (2018).Técnicas conversacionales y narrativas investigación cualitativa con software Nvivo. Editan: Universidad de Granada. Escuela Andaluza de Salud Pública. Recuperado de https://www. researchgate.net/publication/329364863_ Tecnicas_conversacionales_y_narrativas_ Investigacion_cualitativa_con_Software_ NVivo.

Vigotsky, L.S. (1987). Historia del desarrollo de las funciones psíquicas superiores. Ed Científico-Técnica, La Habana.

Zurbano Díaz de Cerio, J. L. (2001). Educación para la convivencia y para la paz. Educación Infantil. Gobierno de Navarra. Departamento de Educación y Cultura. Impresión. Gráficas Ona. Pamplona. España. Recuperado de http://dpto.educacion.navarra.es/ publicaciones/pdf/conv_pri.pdf. Consulta:(19 de noviembre de 2017). 